\title{
Internal Models of Limb Geometry in the Control of Hand Compliance
}

\author{
F. Lacquaniti, N. A. Borghese, and M. Carrozzo \\ Istituto di Fisiologia dei Centri Nervosi, CNR, 20131 Milan, Italy
}

\begin{abstract}
The aim of this article is to describe the role of some neural mechanisms in the adaptive control of limb compliance during preplanned mechanical interaction with objects. We studied the EMG responses and the kinematic responses evoked by pseudorandom perturbations continuously applied by means of a torque motor before and during a catching task. The temporal changes of these responses were studied by means of an identification technique for timevarying systems. We found a transient reversal of EMG stretch reflex responses centered on the time of ball impact on the hand; this reversal results in a transient coactivation of antagonist muscles at both the elbow and the wrist. The kinematic responses describe the relation between torque input and position output. Thus, they provide a global measure of limb compliance. The changes in limb compliance during catching were quantified by computing error criteria either in the Cartesian coordinates of the hand or in the angular coordinates of the elbow and wrist joints. We found that only the hand compliance in Cartesian coordinates is consistently minimized around impact, in coincidence with the transient reversal of the stretch reflex responses. By contrast, the error criteria expressed in the angular coordinates of the joints have a variable time course and are not minimized around impact. It is known that hand compliance depends on both the pattern of muscle activities and the geometrical configuration of the limb. Therefore, the lack of consistent correlation between the changes in hand compliance and the changes in the geometrical configuration of the limb during catching indicates that the gating of the stretch reflex responses around impact time is based on an internal model of limb geometry.
\end{abstract}

Compliant motion of a limb departs from simple positioning insofar as the mechanical interaction with the environment is not rigidly fixed (as, for example, in a constant-gain positionservo), but can be modulated by modifying limb position to accommodate external forces (Mason, 1981; Hogan, 1985). In contrast to most current artificial manipulators, humans exhibit a striking ability to modulate limb compliance according to specific task requirements. ${ }^{1}$ The aim of this article is to describe

\footnotetext{
Received July 19, 1991; revised Oct. 16, 1991; accepted Dec. 6, 1991.

We thank Dr. C. Maioli, J. F. Soechting, and C. Tercuolo for a critical reading of the manuscript, and Mr. L. Chiumiento and F. Neutro for technical help. This work was partially supported by Grant 3149 ESPRIT II Basic Research awarded by the Commission of the European Communities and by a grant awarded by the Human Frontier Science Program.

Correspondence should be addressed to F. Lacquaniti, IFCN, CNR, Via Mario Bianco 9, 20131 Milan, Italy.

Copyright (C) 1992 Society for Neuroscience $0270-6474 / 92 / 121750-12 \$ 05.00 / 0$
}

some new features of the neural mechanisms involved in the adaptive control of limb compliance. We shall argue that these neural mechanisms incorporate accurate internal models of the physical properties of both limbs and objects (cf. Arbib, 1981; Bizzi et al., 1982; Loeb, 1983; Georgopoulos, 1986; Hildreth and Hollerbach, 1987; Atkeson, 1989; Ghez et al., 1990; Soechting and Flanders, 1991).

The problem of the neural mechanisms involved in the control of limb compliance has recently received much attention (Houk, 1979; Feldman, 1980; Humphrey and Reed, 1983; Hogan, 1984; Mussa-Ivaldi et al., 1985; Hasan, 1986). Thus, it has been shown that separate control of joint torque and joint compliance is achieved by means of a variable degree of voluntary coactivation of antagonist muscles (Feldman, 1980; Hogan, 1984). Specific cortical and subcortical centers have been implicated for the control of antagonist coactivation (Humphrey and Reed, 1983; Bizzi et al., 1991).

$\Lambda$ lso, the stretch reflex may contribute to the control of limb compliance. However, the nature of the variable (or variables) controlled by this reflex remains controversial; thus, muscle length, force, or stiffness would be regulated by the reflex loop according to different hypotheses (cf. Houk and Rymer, 1981; Stein, 1982). In particular, the stiffness regulation hypothesis states that the spinal stretch reflex acts to keep the overall muscle stiffness relatively constant under variable operating conditions (Houk, 1979).

Most studies dealing with stretch reflex behavior have involved motor tasks that tend to emphasize positional requirements over compliance requirements. These tasks require the maintenance of a given posture of the limb or the generation of a desired movement in the face of external load perturbations. Under such conditions, the short-latency stretch reflexes evoked by the external perturbations usually obey the law of reciprocal innervation, agonist muscles being activated by the stimulus and antagonists relaxed (Dufresne et al., 1978; Sanes, 1986; Soechting, 1988). Furthermore, the control schemes that have previously been put forward to account for the function of the stretch reflex, involving a negative feedback of joint angular position, velocity, or torque, are all predicated on the law of reciprocal innervation (cf. Agarwal and Gottlieb, 1984; Soechting and Lacquaniti, 1988).

\footnotetext{
Compliance is defined as the relation between an applied force and the resulting displacement (its inverse is the impedance). It includes both static components (e.g., stiffness) and dynamic components (e.g., viscosity). Limb compliance can be equated to the resistance opposed to an external force. This resistance is determined by limb inertia and restoring forces, such as those that result from the intrinsic viscoelastic properties of muscles and tendons and from muscle contractions of voluntary or reflex origin.
} 
Only recently has it been possible to investigate the behavior of the stretch reflex during the mechanical interaction with objects and during their manipulation. One might guess that the functional demands of compliant motion posed by the mechanical interaction call for a specific mode of reflex control that is different from the reflex mode classically described in positioning tasks. This has indeed been found to be the case experimentally. Thus, during the task of catching a free-falling ball, the reflex responses evoked by ball impact on the hand violate the law of reciprocal innervation, since flexor and extensor muscles of wrist and elbow joints are coactivated at a short latency (Lacquaniti and Maioli, 1987, 1989a,b). A similar behavior has been obtained in response to sudden load changes during precision grip of objects (Traub et al., 1980; Cole and Abbs, 1988; Johansson and Westling, 1988). It has also been shown that reflex coactivation is not solely contingent on the occurrence of adequate peripheral stimuli, but can be preset within the CNS by switching from the spinal pathways of reciprocal inhibition to those of coactivation of antagonist $\alpha$-motoneurons (Lacquaniti and Maioli, 1987, 1989a; Lacquaniti et al., 1991).

These studies have clearly indicated that reflex coactivation represents a distinct mode of operation that the CNS can turn on during brief, selective periods of time, in coincidence with specific forms of mechanical interaction of the hand with objects. The question then arises quite naturally of what is the function of reflex coactivation vis-a-vis the problem of compliant motion during mechanical interaction. Catching a moving ball is an ideal paradigm for the assessment of the functional significance of different modes of motor control. In fact, this task requires a control of limb position to intercept ball trajectory. In parallel, it also requires a control of limb compliance to absorb ball momentum during the dynamic interaction between the ball and the hand. Moreover, both the control of limb position and the control of limb compliance must comply with strict timing constraints in order to be successful (Sharp and Whiting, 1974; Lacquaniti and Maioli, 1989a).

The experiments described in this article were designed to address the issue of the role that different neural mechanisms may have in the control of the position and compliance of the limb. We studied the EMG responses and the kinematic responses evoked by pseudorandom perturbations continuously delivered by means of a torque motor before and during catching. The temporal changes of these responses during the catching task were studied by means of an identification technique for time-varying systems (Soechting et al., 1981; Lacquaniti et al., 1982b).

Since the responses of the kinematic variables describe the relation between torque input and position output, they provide a measure of the compliance of the limb (Agarwal and Gottlieb, 1977; Hunter and Kearney, 1982; Lacquaniti et al., 1982b; Kearney and Hunter, 1990). In order to dissociate the relative role played in the control of compliance by stretch reflex responses from the role played by overall muscle activity and limb position, we compared the time course of the changes during catching in each such variable with that of the changes in limb compliance.

The analysis of the kinematic responses was also aimed at addressing the so-called "inverse problem" of optimal control: given a dynamical system, what is the goal for which the control law is optimal (Hogan, 1984; Stein et al., 1988)? Two specific hypotheses were contrasted: (1) the goal of the neuromuscular

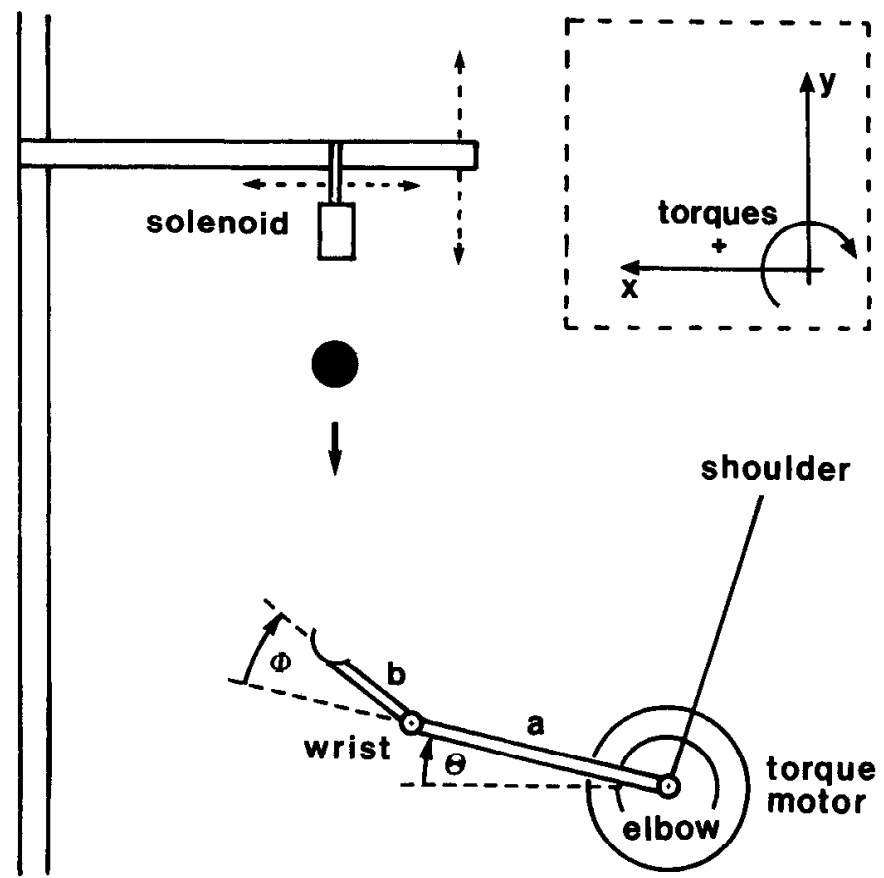

Figure 1. Schematic of the experimental setup. Forearm and hand were strapped to an electrogoniometer with two degrees of freedom, which measured elbow $(\Theta)$ and wrist $(\Phi)$ angles. The elbow joint was aligned with the shaft of a torque motor. Subjects kept their hand fully supinated and wore a stiff glove. The ball was dropped by the solenoid whose horizontal position was adjusted so that, with the forearm and hand horizontal, the mcp of the middle finger was on the line of fall. The inset describes the sign convention for the joint torques and the Cartesian coordinates of the hand.

control is to minimize around the time of ball impact the oscillations of the joints in angular coordinates; (2) the system minimizes around impact the oscillations of the hand in world (c.g., Cartesian) coordinates. In a multijointed limb, these two sets of variables need not covary. In fact, we consistently found that only hand compliance in world coordinates is effectively minimized around impact, in coincidence with transient reversal of the stretch reflex responses. Since hand compliance is related to the dynamical state of the whole limb, we suggest that its minimization is based on an internal model of the geometry of the limb.

A brief account of these results has appeared in abstract form (Lacquaniti et al., 1990).

\section{Materials and Methods}

Experimental setup and protocol. The general experimental procedures have been described in detail in a previous article (Lacquaniti et al., 1991). Bricfly, subjects were instructed to catch a ball $(600 \mathrm{gm}, 9 \mathrm{~cm}$ in diameter) dropped from $1.6 \mathrm{~m}$ (Fig. 1). Their right arm, fully supinated, was strapped to a goniometer that measured the angle of flexionextension at the elbow $(\Theta)$ and wrist $(\Phi)$. Movement was constrained to the vertical plane by the apparatus. The metacarpophalangeal joint (mcp) of the middle finger was on the ball's line of fall. The EMG activities of biceps, brachioradialis, triceps, flexor carpi radialis (FCR), flexor carpi ulnaris (FCU), extensor carpi radialis (ECR), and extensor carpi ulnaris $(E C U)$ were recorded by means of surface electrodes.

Trains of torque pulses with either positive or negative polarity of a pseudorandom binary sequence (PRBS) were applied continuously during each trial ( $2.56 \mathrm{sec}$ duration) by means of a torque motor coupled to the elbow joint. The ball was dropped 1 sec after trial start. The sixthorder maximum-length sequence consisted of positive and negative pulscs. Pulsc amplitude was $10 \mathrm{~N} \cdot \mathrm{m}$ peak to peak, and the duration varied pseudorandomly from 20 to $120 \mathrm{msec}$ in integer multiples of 20 

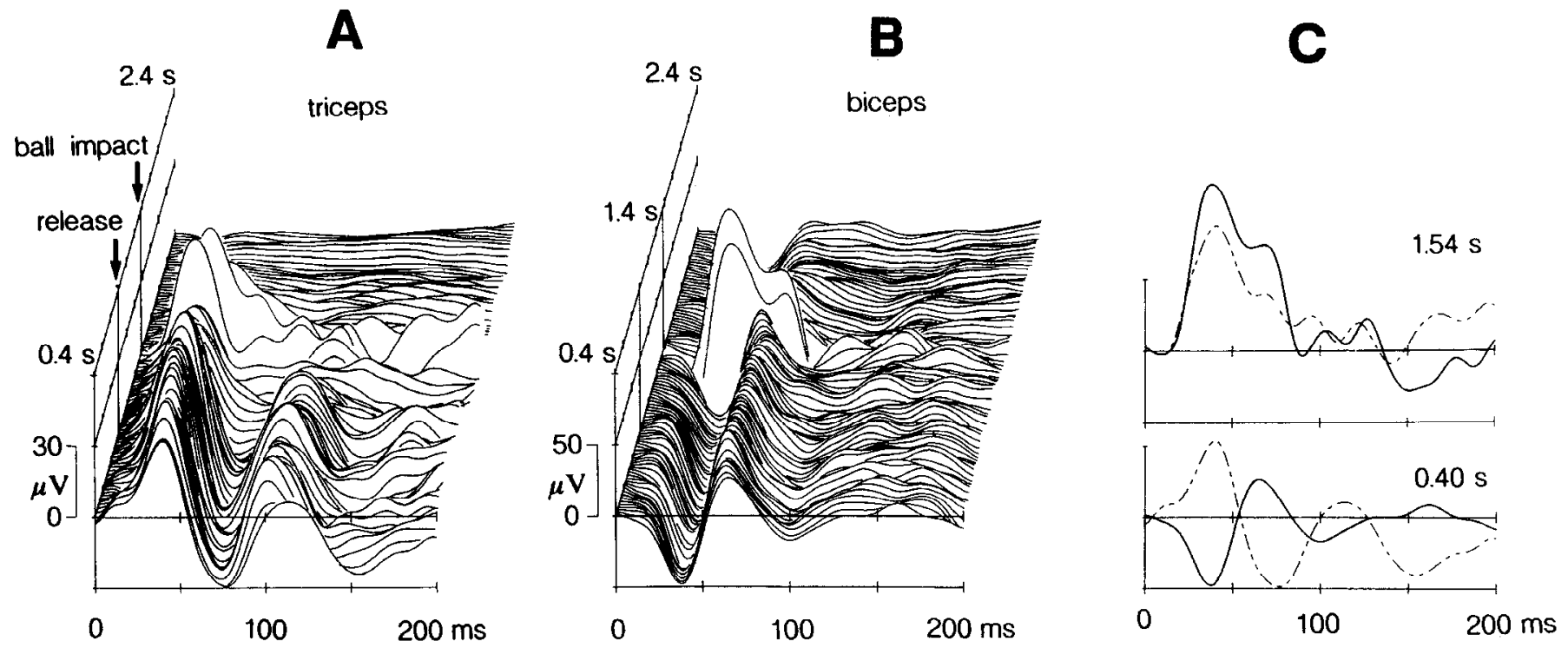

Figure 2. Impulse responses of triceps and biceps EMG activity from one experiment. These responses were obtained by cross-correlating the EMG activities with the pseudorandom perturbations. In $A$ and $B$, each trace is the EMG response at the time indicated by the oblique scale, time being measured from the onset of the perturbations. As plotted, the responses represent the average contribution to the motor output by a 20 msec torque pulse tending to flex the elbow and occurring $0-200 \mathrm{msec}$ before. The vertical lines denote the time of release (1 sec) and impact of the ball on the hand $(1.55 \mathrm{sec}) . C$. The impulse responses of biceps (solid traces) and triceps (broken traces), obtained at 0.4 sec (lower panel) and at 1.54 sec (upper panel), are superimposed. Note the reversal of biceps responses around impact time.

msec. The sequence was shifted by one element from trial to trial until all 63 elements had been shifted. This procedure was repeated four times ( 252 trials) to increase the signal-to-noise ratio. All experiments were totally harmless for the subjects.

Data analysis. Kinematic and EMG data were sampled by a computer at a rate of $1000 / \mathrm{sec}$. Sixty-three averages were constructed (after fullwave rectification of the EMG activities) by including all trials with a corresponding PRBS run. The Cartesian coordinates $(x, y)$ of the third metacarpophalangeal joint were computed trigonometrically from measured elbow $(\Theta)$ and wrist $(\Phi)$ angles:

$$
\begin{aligned}
& x=a \cos \Theta+b \cos (\Theta+\Phi), \\
& y=a \sin \Theta+b \sin (\Theta+\Phi),
\end{aligned}
$$

where $a$ and $b$ are the length of the forearm and third metacarpus, respectively (see Fig. 1). The time-varying impulse responses of each output (EMG activities and kinematic variables) to a $20 \mathrm{msec}$ torque pulse were computed at 20 msec intervals by cross-correlating the PRBS input with the outputs across all 63 averages. The impulse responses were computed over a $400 \mathrm{msec}$ interval from pulse onset. They accounted well for the data, since the output predicted by the convolution with PRBS was highly correlated with the actual output (on average, the correlation coefficient was 0.80 for the EMG activities and 0.94 for the kinematic variables).

Because the impulse responses of the kinematic variables describe the relation between torque input and position output, they provide a nonparametric measure of the compliance of the limb (Hunter and Kearney, 1982; Lacquaniti et al., 1982b; Kearney and Hunter, 1990). The smaller the magnitude of the oscillations of the kinematic responses, the smaller the limb compliance, and vice versa. Consequently, the changes of limb compliance during catching can be quantified by computing the global amount of mechanical oscillations resulting from a torque pulse applied at each different time during the task. As global measures of oscillation, we computed a number of error criteria that are widely used as performance index for adaptive control (Davies, 1970; Aström and Wittenmark, 1989). These criteria correspond to the time integral of the values of kinematic impulse responses. Their discrete-time version is computed as the sum of squared deviations from the mean value,

$$
E_{1}=\sum(v-\hat{v})^{2},
$$

as the sum of absolute values,

$$
E_{2}=\sum|v|,
$$

or as the sum of time-weighted absolute values

$$
E_{3}=\sum t|v| \text {. }
$$

In Equations 3-5, $v$ corresponds to $\Theta, \Phi, x$, or $y, \hat{v}$ is their respective mean value, and $t$ is time. $E_{3}$ puts a greater penalty than $E_{1}$ or $E_{2}$ on oscillations that are poorly damped. A further error criterion $\left(E_{4}\right)$ was defined as the area of the $95 \%$ confidence ellipsoid of the $(x, y)$ coordinates of the hand (Georgopoulos et al., 1981). For each impulse response obtained at a different time after the onset of the perturbations, an error criterion was computed by summing over the entire $400 \mathrm{msec}$ interval of definition of the impulse response.

\section{Results}

\section{Impulse responses of EMG activity}

Figure 2 shows the impulse responses of triceps $(A)$ and biceps $(B)$ EMG activity obtained in one experiment. The oblique axis represents time $\left(t_{j}\right)$ measured from the onset of the PRBS, and the arrows indicate the time of ball release (at $1 \mathrm{sec}$ ) and impact on the hand (at $1.55 \mathrm{sec}$ ). Each trace corresponds to the average response at $t_{j}$ to a $20 \mathrm{msec}$ torque pulse tending to flex the elbow. The temporal resolution of $t_{j}$ is $20 \mathrm{msec}$.

The waveform of these EMG impulse responses has been described extensively elsewhere (Lacquaniti et al., 1991). Here, we will briefly recapitulate the changes of the responses during the catching task. The pattern of the short-latency (about 20 msec) EMG responses obeyed the law of reciprocal innervation of antagonist muscles at any time during the trial, except during a limited time interval centered on the time of ball impact. During that interval (from about $60 \mathrm{msec}$ prior to impact up to about $70 \mathrm{msec}$ after impact), the pattern of the responses consisted of a substantial coactivation of both stretchcd and shortening muscles. This is demonstrated in Figure $2 C$ : biceps responses (solid traces) and triceps responses (broken traces) obtained at $0.4 \mathrm{sec}$ (basal conditions) and at $1.54 \mathrm{sec}(10 \mathrm{msec}$ 

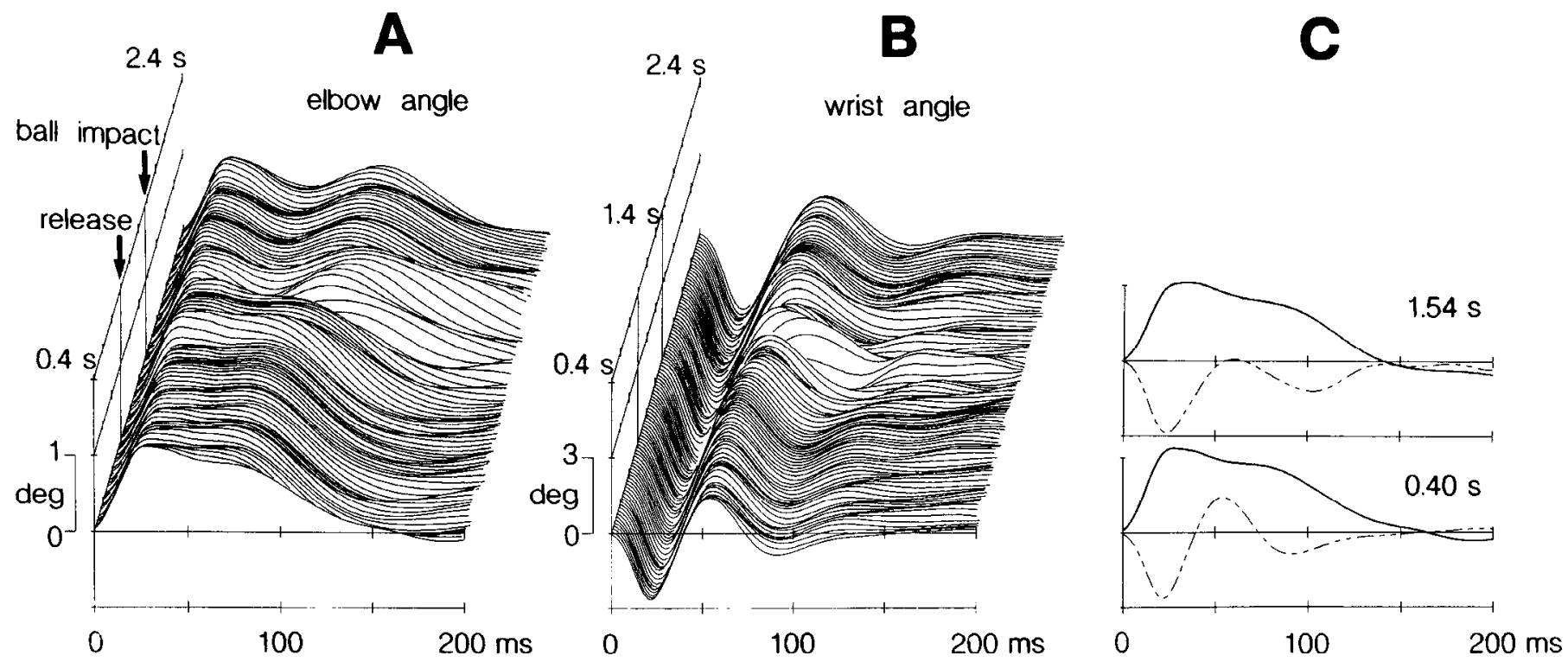

Figure 3. Impulse responses of elbow angle and wrist angle from one experiment plotted in the same format as in Figure 2. Changes in flexion of joint angles are positive (see Fig. 1). C. The impulse responses of elbow angle (solid traces) and wrist angle (broken traces), obtained at 0.4 sec (lower panel) and at $1.54 \mathrm{sec}$ (upper panel), are superimposed.

before impact) are plotted in the lower and upper parts, respectively. Reflex coactivation resulted from a transient reversal of the direction of the short-latency responses of flexor muscles (biceps and brachioradialis), with limited changes of triceps responses. Reversal of flexor responses began $63 \pm 32( \pm \mathrm{SD})$ msec before impact and ended $70 \pm 38$ msec after impact. A similar coactivation of the reflex responses of flexor and extensor muscles of the wrist also occurred within a similar time epoch (Lacquaniti et al., 1991).

\section{Impulse responses of the kinematic variables}

Limb kinematics during catching can be described in terms of either the changes of elbow $(\Theta)$ and wrist $(\Phi)$ angles or the changes of hand position in Cartesian space. Hand position is defined by the $(x, y)$ coordinates of the third mcp, where the impact of the ball occurs (see Fig. 1). The changes of elbow and wrist responses to the perturbations during the catching task were rather subtle. However, their combined effect resulted in clearcut changes of hand position responses: the vertical oscillations of the hand decreased substantially around impact time.

Figure 3 shows the impulse responses of elbow $(A)$ and wrist $(B)$ angles obtained in one experiment, plotted in the same format as in Figure 2. Both elbow and wrist angle responses are oscillatory. A positive pulse applied to the elbow joint resulted in an initial flexion of the elbow and an initial extension of the wrist because of the dynamic mechanical coupling between the two joints. The period of the damped oscillations is longer at the elbow than at the wrist, mainly due to the larger inertia. For the sake of comparison, elbow responses (solid traces) and wrist responses (broken traces) are superimposed in Figure $3 C$. The responses obtained at $0.4 \mathrm{sec}$ and at $1.54 \mathrm{sec}$ are plotted in the lower and upper panel, respectively.

Elbow responses consisted of a double-peaked positive wave followed by a much smaller undershoot of the baseline. Neither the first positive peak (at about $50 \mathrm{msec}$ from pulse onset) nor the second positive peak (at about $100 \mathrm{msec}$ ) changed substantially during the first part of the task, up to about $1.8 \mathrm{sec}$. Only toward the end of the trial did the second peak become slightly more pronounced and prolonged. Also, the first peak in extension of wrist responses (at about $25 \mathrm{msec}$ ) did not change appreciably, but the subsequent overshoot in flexion (at about 60$70 \mathrm{msec}$ ) was reduced in magnitude and shifted to longer latencies after impact.

Figure 4 shows the changes in the horizontal $(A)$ and vertical (B) responses of hand position for the same experiment as Figure 3 . The perturbations produced significant oscillations only in the vertical direction, but not in the horizontal. ${ }^{2}$ The combined effect of elbow and wrist angular motions resulted in an upward movement of the hand that peaked around $50 \mathrm{msec}$ from pulse onset and was generally damped out within $200 \mathrm{msec}$. The peak amplitude of this vertical oscillation was much smaller around impact time than before or after it. Thus, the peak amplitude at $1.54 \mathrm{sec}$ is $63 \%$ of that at $0.4 \mathrm{sec}$ in Figure $4 C$.

The overall changes of the geometrical configuration of the limb that are due to the PRBS perturbations, on one side, and to the movement associated with catching, on the other side, can be appreciated in Figure 5. Each series of stick diagrams depicts the limb trajectory over the $400 \mathrm{msec}$ interval from pulse onset, as derived from the kinematic impulse responses (thc data are from an experiment different from that of Figs. 3 and 4). The series of limb trajectories obtained at $20 \mathrm{msec}$ intervals have been offset along the vertical in Figure 5 (see the indicated time after the onset of the trial). The corresponding values of elbow and wrist angles derived from the ensemble average have been added to each impulse response. Thus, one can note the

\footnotetext{
${ }^{2}$ This is due to the mean configuration of the limb during the task. The hand never deviated by more than $10 \mathrm{~cm}$ from the horizontal (see stick diagrams in Fig. 9). Initially (prior to ball release), the forearm was slightly flexed relative to the horizontal $\left(\theta=12^{\circ} \pm 4^{\circ}\right.$, mean \pm SD over eight experiments).
} 


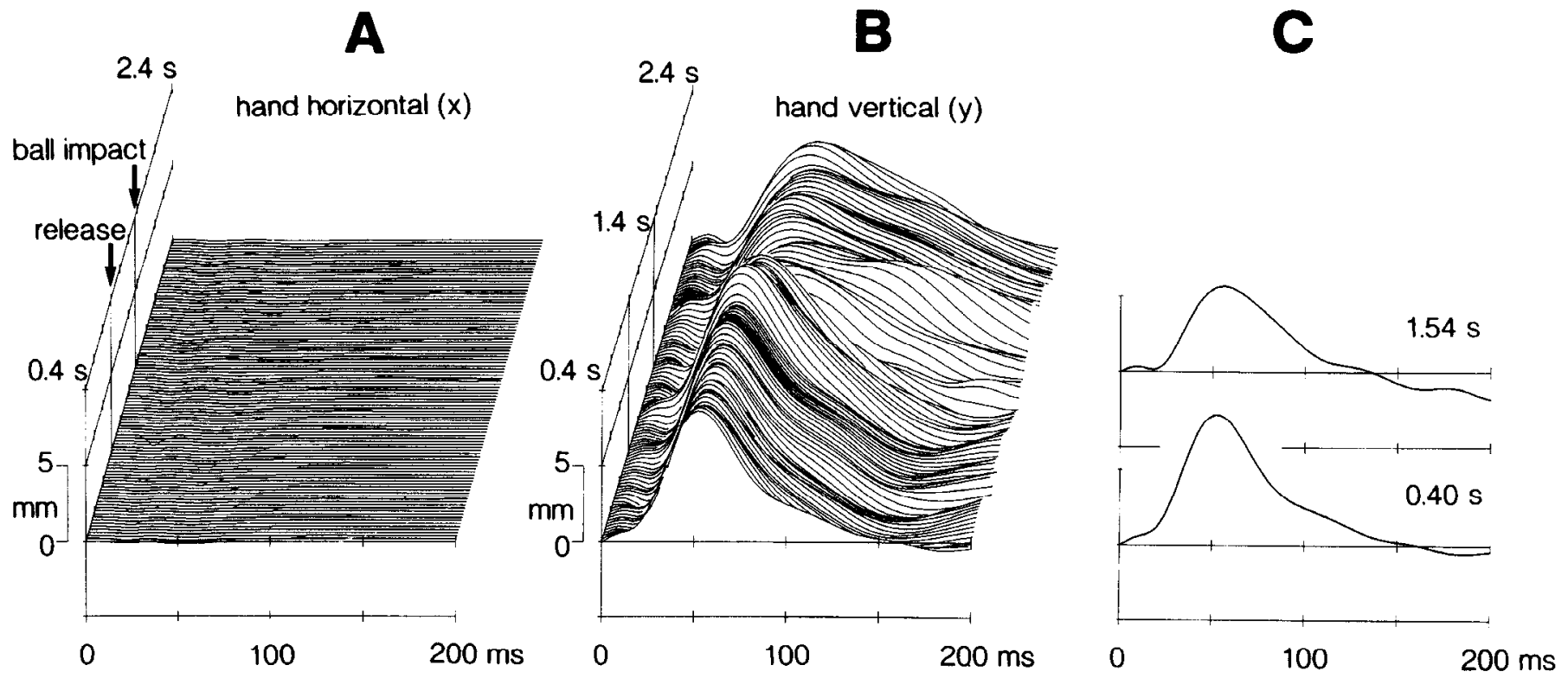

Figure 4. Impulse responses of horizontal $(x)$ hand position and vertical $(y)$ hand position from the same experiment as that of Figure 3. Upward $y$ changes are positive (see Fig. 1). $C, y$ impulse responses obtained at $0.4 \mathrm{sec}$ and at $1.54 \mathrm{sec}$ are plotted in the lower and upper panels, respectively. Note the decrement of $y$ responses around impact time.

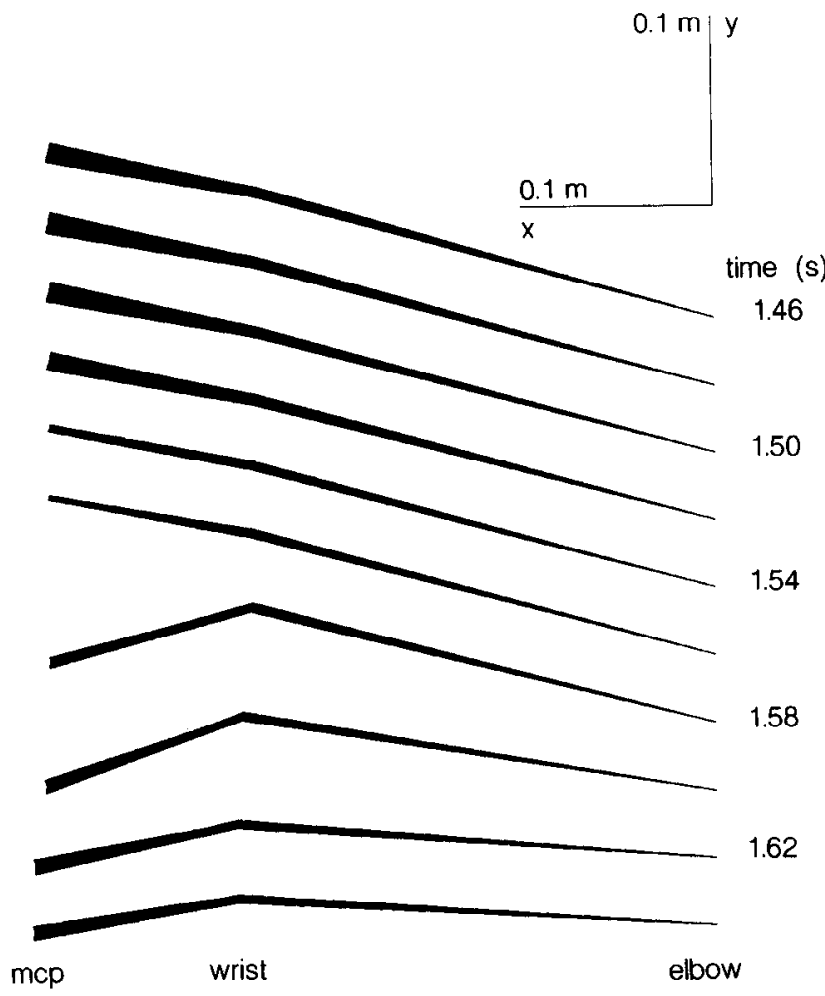

Figure 5. Overall changes of the geometrical configuration of the limb that are due to the PRBS perturbations, on one side, and to the movement associated with catching, on the other side, in one experiment. Each series of stick diagrams depicts the limb trajectory over the 400 msec interval from pulse onset, as derived from the kinematic impulse responses. The corresponding values of elbow and wrist angles derived from the ensemble average have been added to each impulse response. The resulting series of limb trajectories obtained at $20 \mathrm{msec}$ intervals have been offset along the vertical; the corresponding time after trial onset is indicated on the right. The thickness of each series of stick diagrams indicates the amount of spatial excursion of the corrcsponding joints. upward flexion of the limb prior to ball impact (occurring at $1.55 \mathrm{sec}$ ) and the downward extension after impact.

The thickness of each series of stick diagrams indicates the peak-to-peak spatial excursion of the corresponding joints. The thickness at mcp is much smaller in the series at $1.54 \mathrm{sec}$ and $1.56 \mathrm{sec}$ than that in earlier or later series. Note, however, that the corresponding thickness at the wrist is not very different in all such cases, due to the limited variations in the amplitude of the elbow angular motion due to PRBS during catching.

\section{Time course of the changes in limb compliance during catching}

The compliance of the limb is defined nonparametrically by the impulse responses of the kinematic variables described above. The smaller the magnitude of the oscillations of the kinematic responses, the smaller the limb compliance, and vice versa. To quantify the changes of limb compliance during catching, we computed error criteria for each impulse response obtained at a different time during the task. Figure 6 illustrates the time courses of the changes in the integral of squared error $\left(E_{1}\right.$ in Eq. 3 of Materials and Methods). [The results obtained using the other error criteria examined $\left(E_{2}-E_{4}\right)$ were very similar to those with $E_{1}$.] The traces correspond to the grand averages of the results obtained in all experiments and subjects $(n=8)$.

Figure $6 \mathrm{~A}$ shows the time course of the error of the impulse responses of elbow angle [trace labeled $\Sigma(\Theta)^{2}$ ] and wrist angle $\left[\Sigma(\Phi)^{2}\right]$. The changes of these errors are complex and multiphasic around impact time. For instance, the error at the wrist has a minimum $30 \mathrm{msec}$ prior to impact, followed by a maximum 30 msec after impact and by a second minimum. However, the value at impact is not significantly different from the basal value, at either the wrist or the elbow.

Figure $6 B$ shows the time course of the error of the horizontal $\left[\Sigma(x)^{2}\right]$ and vertical $\left[\Sigma(y)^{2}\right]$ responses of hand position. The former is very close to, and not distinguishable from, the zero line, since the horizontal oscillations induced by PRBS were negli- 

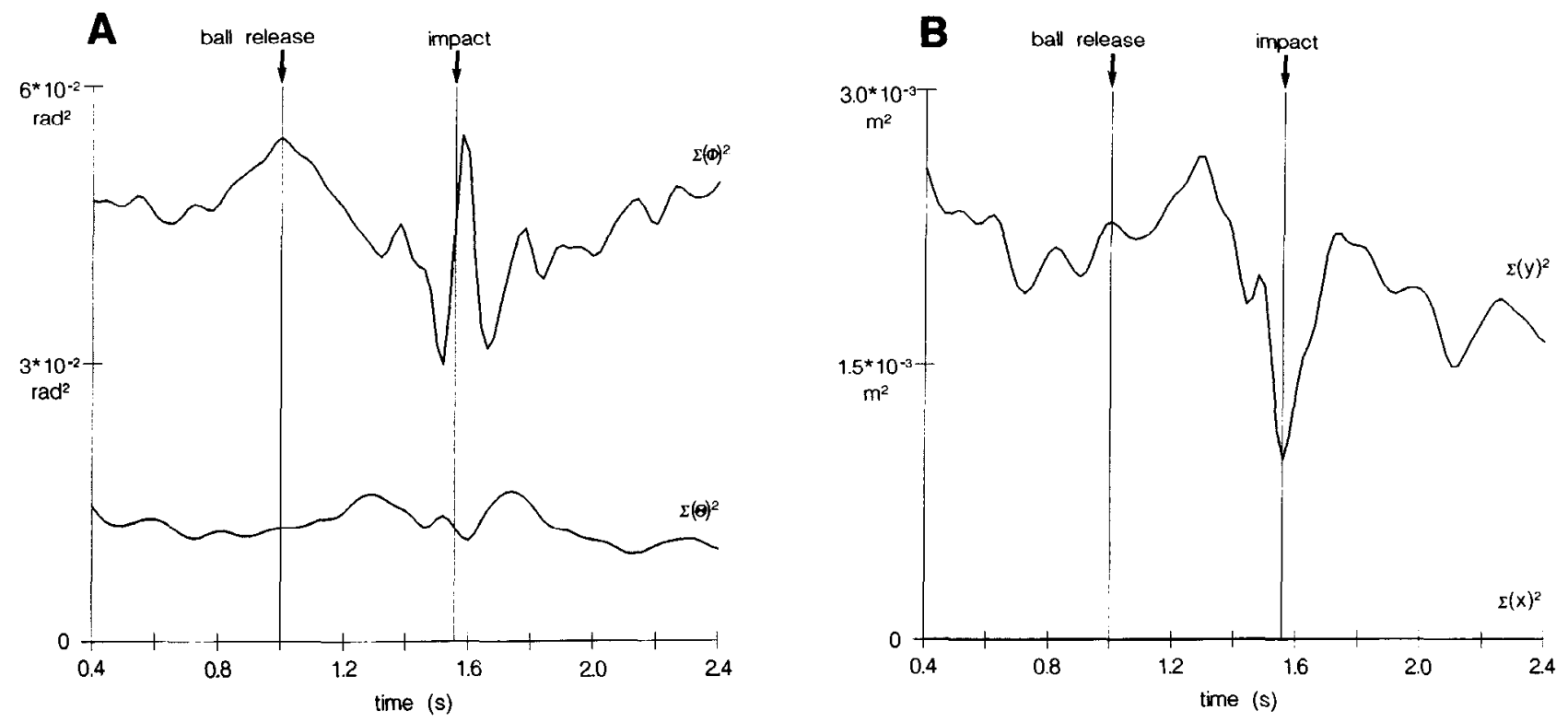

Figure 6. Time course of the changes in the integral of squared errors of kinematic impulse responses. The traces correspond to the grand averages of the results obtained in all experiments and subjects. $A$ : Top trace, error of wrist responses $\left[\Sigma(\Phi)^{2}\right]$; bottom trace, error of elbow responses $\left[\Sigma(\Theta)^{2}\right]$. $B$ : Top trace, error of vertical responses of hand position [ $\left.\Sigma(y)^{2}\right]$; bottom trace, crror of horizontal responses [ $\left.\Sigma(x)^{2}\right]$. This lattcr trace is very close to the zero line, since the horizontal oscillations induced by PRBS were negligible (see Fig. $4 A$ ). Note the sharp minimum of $\Sigma(y)^{2}$ centered on impact time. The vertical lines denote the time of ball release and the time of ball impact on the hand.

gible (see Fig. 4A). The latter has a clear-cut modulation with a steep valley centered on impact: it decreases significantly below the baseline $(99 \%$ confidence limits of the mean value computed over the $0.4-1.0 \mathrm{sec}$ interval) starting from $30 \mathrm{msec}$ prior to impact, reaches the minimum at impact, and returns within the confidence limits of the baseline $130 \mathrm{msec}$ after impact. On average, the minimum error occurs at $2 \pm 6 \mathrm{msec}$ (mean \pm SD over all experiments) after the impact; its value is $45 \pm 13 \%$ of the baseline.

\section{Temporal correlation between the changes in limb compliance and the changes in muscle activity}

The overall compliance of the limb is determined by the specific operating point. In particular, it depends on perturbation amplitude, limb position, mean EMG activity, and reflex behavior (cf. Kearney and Hunter, 1990). In the present experiments, the amplitude of PRBS perturbations was constant throughout the task. However, the other parameters did change. Thus, in order to disassociate their relative role, in each experiment we compared their time course with that of the changes in limb compliance in search of consistent correlations.

Each panel of Figures 7 and 8 shows the results from a different experiment (and subject). The top two traces correspond to the changes in the errors of elbow responses $\left[\Sigma(\Theta)^{2}\right]$ and wrist responses $\left[\Sigma(\Phi)^{2}\right]$. Their time course is complex and somewhat variable among experiments. By contrast, the time course of the changes in the error of the vertical responses of hand position $\left[\Sigma(y)^{2}\right.$, third trace from the top] is roughly similar in all experiments and corresponds to that previously described, with the minimum near impact time.

This time course parallels closely that of the changes in the stretch reflex responses evoked by PRBS. The fourth trace from the top corresponds to the mean amplitude of the short-latency EMG responses of biceps muscle in Figures $7 A$ and $8, A$ and $B$ (labeled biceps reflex), and extensor carpi ulnaris in Figure $7 B$ (ECU reflex). As previously described, a reversal of the direction of reflex responses was consistently observed in all experiments within the time interval of about $60 \mathrm{msec}$ prior to impact up to about $70 \mathrm{msec}$ after impact. These changes in stretch reflex behavior are therefore well correlated with those in hand compliance.

By contrast, the time course of the overall EMG activity (dashed envelopes in Figs. 7,8 ) is poorly correlated with the changes in hand compliance. EMG profile is multiphasic. In general, anticipatory activity built up prior to impact (as in Fig. $7 A, B)$. However, the EMG baseline prior to impact was rather variable among different muscles and experiments. Thus, in most cases the EMG amplitude at impact was greater than under basal conditions (0.4-1.0 sec interval). However, in some cases (17\% of all cases) it was lower, for instance, in triceps in Figure $7 A$, biceps and triceps in Figure $8 A$, and triceps and ECU in Figure $8 B$. This variability depends in part on the different strategy adopted by the subjects: some subjects (e.g., in Fig. $7 B$ ) started off with a moderate amount of muscle activity and built up activity just prior to impact, while others (e.g., in Fig. $8 A, B$ ) began by resisting strongly the PRBS perturbations and subsequently reduced their muscle activity in order to catch the ball effectively.

A transient increase of EMG activity much larger than the anticipatory activity was reflexly elicited by the impact. These reflex responses peaked 40-60 msec after impact. Therefore, they cannot account for the minimum in hand compliance at impact.

\section{Correlation between the changes in limb compliance and the changes in limb geometry}

It has been shown that, when subjects maintain a static limb posture, the hand stiffness depends strongly on the location of 

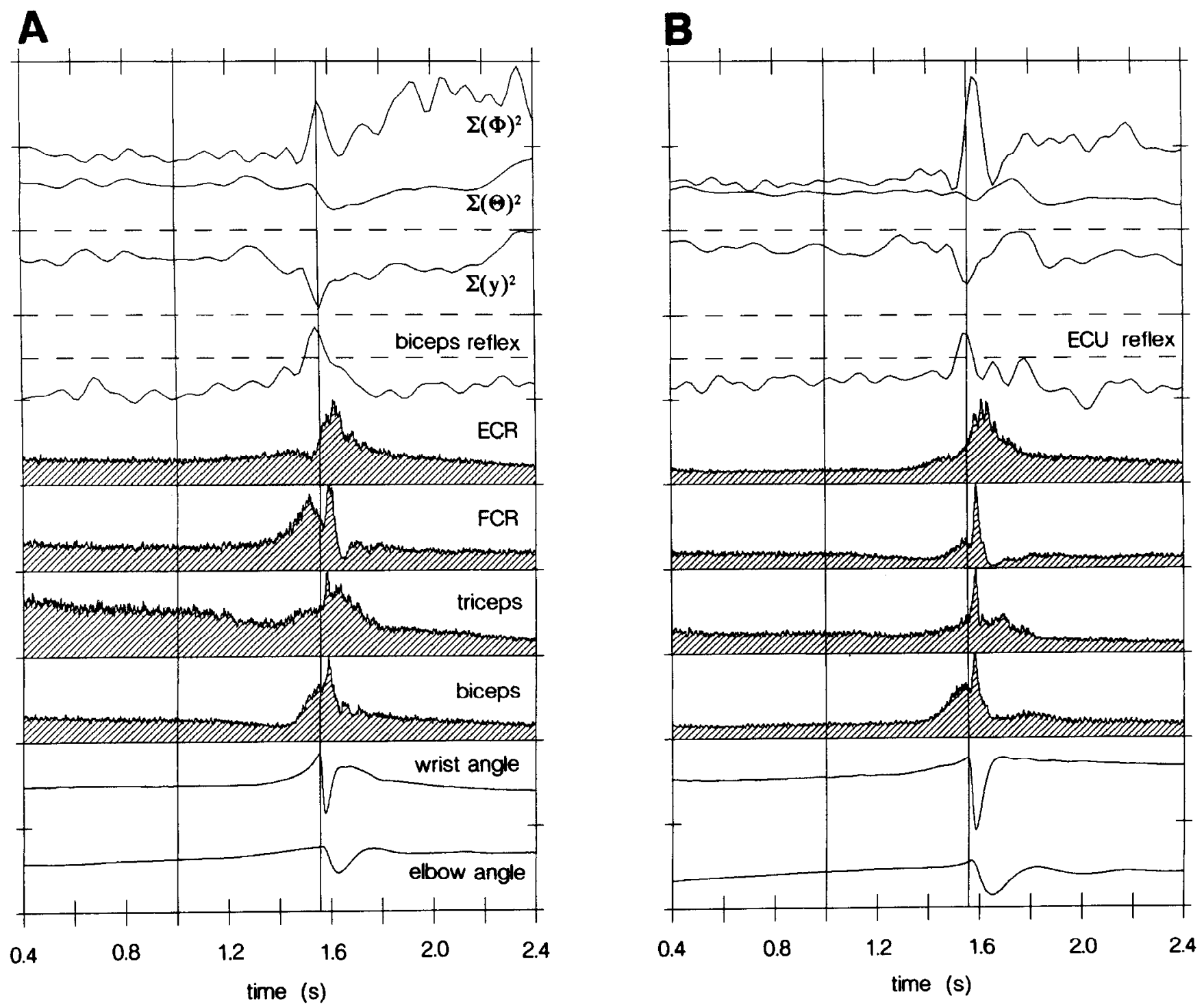

Figure 7. Temporal correlation between the changes in limb compliance and the changes in muscle activity. The results from two different experiments are plotted in $A$ and $B$, respectively. The vertical lines denote the time of ball release and impact on the hand. Traces from top to bottom correspond to error of wrist responses $\left[\Sigma(\Phi)^{2}\right]$; error of elbow responses $\left[\Sigma(\Theta)^{2}\right]$; error of vertical hand responses [ $\left.\Sigma(y)^{2}\right]$; mean amplitude of biceps $(A)$ or ECU $(B)$ responses; rectified EMG activity of ECR, FCR, triceps, and biceps; wrist angle $(\Phi)$; and elbow angle $(\Theta)$. The six bottom traces are derived from the ensemble average. The mean amplitude of biceps reflex $(A)$ has bcen computcd over the $20-60$ mscc interval from pulse onset; the mean amplitude of ECU reflex $(B)$, over the $20-40 \mathrm{msec}$ interval. Zero lines for $\Sigma(\Phi)^{2}, \Sigma(\Theta)^{2}, \Sigma(y)^{2}$, and reflex amplitude are broken horizontal lines. Note the reversal of the direction of the mean reflex response in both biceps and ECU. In $A$, scales (per division) are as follows: $0.02 \operatorname{rad}^{2}\left[\Sigma(\Phi)^{2}, \Sigma(\Theta)^{2}\right], 0.0025 \mathrm{~m}^{2}\left[\Sigma(y)^{2}\right], 120 \mu \mathrm{V}$ (biceps reflex), $30^{\circ}(\Phi, \Theta)$. In $B$, scales are as follows: $0.035 \mathrm{rad}^{2}\left[\Sigma(\Phi)^{2}, \Sigma(\Theta)^{2}\right], 0.0027 \mathrm{~m}^{2}\left[\Sigma(y)^{2}\right]$, $80 \mu \mathrm{V}(\mathrm{ECU}$ reflex $), 30^{\circ}(\Phi, \Theta)$. Rectified EMG traces of ECR, FCR, triceps, and biceps have been scaled to their maximum.

the hand in the external space; in other words, hand stiffness varies systematically with the geometrical configuration of the limb (Mussa-Ivaldi et al., 1985; Flash and Mussa-Ivaldi, 1990). Therefore, one could expect that the values of hand compliance correlated consistently with the instantaneous geometrical configuration of the limb also during the catching task. Lack of correlation would instead indicate that the changes in limb geometry are taken into account and compensated for by the CNS.

The time course of the changes in joint angles during catching can bc appreciated scparately for the elbow and the wrist joints in Figures 7 and 8 (the two bottom traces). Anticipatory activity was often associated with flexion of both elbow (on average, $5^{\circ}$ $\left.\pm 1^{\circ} ; n=5\right)$ and wrist $\left(10^{\circ} \pm 5^{\circ} ; n=6\right)$ preceding impact (as in the case of Fig. $7 A, B$ ). However, this anticipatory movement was somewhat variable among experiments; thus, there was extension (rather than flexion) of the wrist in Figure $8 \mathrm{~A}$ and of the elbow in Figure $8 B$ (extension of one or the other joint occurred in $31 \%$ of all cases). Ball impact on the hand resulted in a brisk extension of wrist and elbow, damped out within about 300 msec.

Hand position in external space depends on the overall geometrical configuration of the limb and cannot be appreciated directly from plots such as those of Figures 7 and 8 . However, the changes in hand compliance can be correlated spatially with 

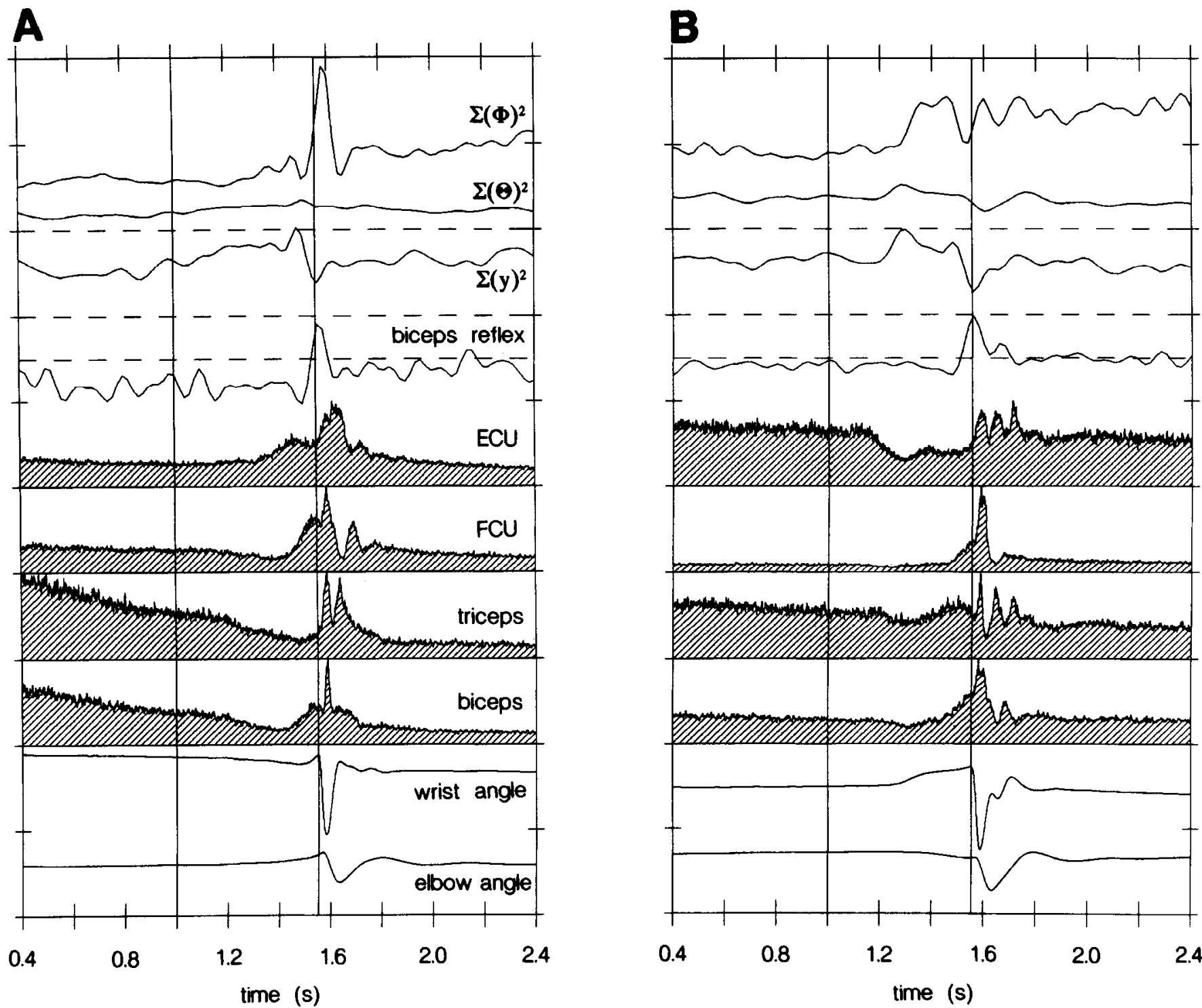

Figure 8. Temporal correlation between the changes in limb compliance and the changes in muscle activity in two experiments different from those of Figure 7 (same format as in Fig. 7). In $A$, scales (per division) are as follows: $0.045 \operatorname{rad}^{2}\left[\Sigma(\Phi)^{2}, \Sigma(\Theta)^{2}\right], 0.0023 \mathrm{~m}^{2}\left[\Sigma(y)^{2}\right], 80 \mu \mathrm{V}($ biceps reflex), $30^{\circ}(\Phi, \theta)$. In $B$, scales are as follows: $0.025 \mathrm{rad}^{2}\left[\Sigma(\Phi)^{2}, \Sigma(\theta)^{2}\right], 0.0018 \mathrm{~m}^{2}\left[\Sigma(y)^{2}\right], 80 \mu \mathrm{V}$ (biceps reflex), 30 $(\Phi, \theta)$. Rectified EMG traces of ECR, FCR, triceps, and biceps have been scaled to their maximum.

the changes in hand position by inspecting Figure 9. The stick diagrams depict the trajectory of the limb from $200 \mathrm{msec}$ prior to impact up to $80 \mathrm{msec}$ after impact, as derived from the ensemble averages. The experiments are numbered sequentially. In each experiment, the stick diagram corresponding to impact is marked with an asterisk placcd to the left and at the same height as the mcp of the hand. In order to correlate spatially the changes in hand compliance with the changes in hand position, the former are plotted on the left of the corresponding set of stick diagrams. As before, hand compliance is globally estimated as the error of the vertical impulse responses of hand position $\left[\Sigma(y)^{2}\right]$. In each experiment, the error function is plotted with the value of $\Sigma(y)^{2}$ on the $x$-coordinate and the value of the corresponding hand vertical coordinate on the $y$-coordinate. $\Sigma(y)^{2}$ has been normalized between the two broken vertical lines, the maximum value being aligned on the leftmost line and the minimum value on the rightmost line. As in the case of the stick diagrams, the error function is plotted over the time interval from $200 \mathrm{msec}$ prior to impact up to $80 \mathrm{msec}$ after impact. Time progresses in the direction indicated by the arrows. For instance, in experiment 2, hand compliance starts from a maximum at $200 \mathrm{msec}$ prior to impact, drops to a minimum at impact time, and increases again afterwards. In that same experiment, the hand starts off slightly higher than the zero line on the vertical, is slightly raised prior to impact (when the stick diagrams are closely clustered together), and is rapidly deviated downward after impact (as indicated by the wide fanning out of the stick diagrams).

In general, the plots of Figure 9 show that the changes in hand compliance are poorly correlated with the changes in hand po- 

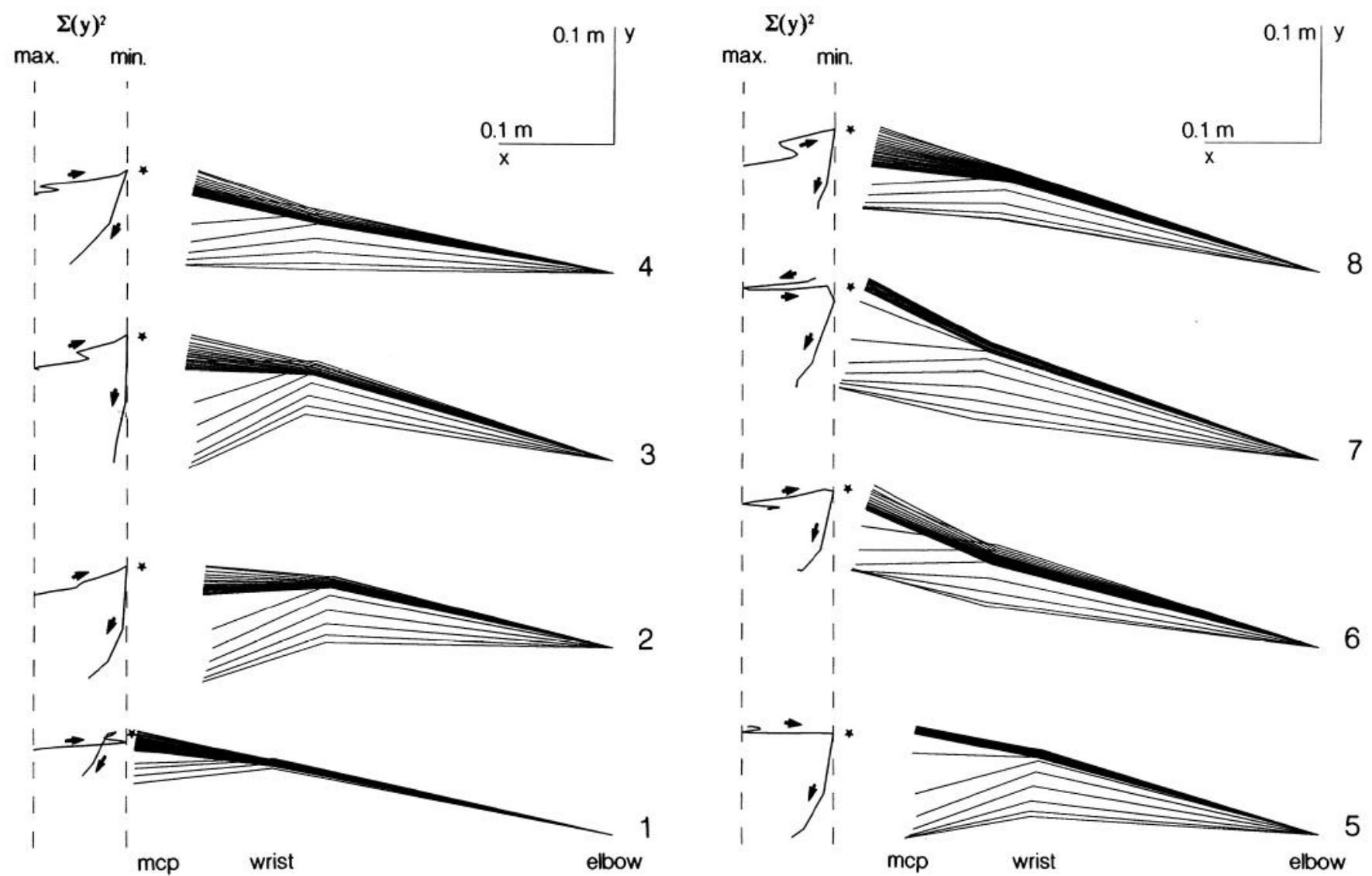

Figure 9. Correlation between the changes in limb compliance and the changes in limb geometry in all eight experiments. For each experiment, the stick diagrams depict the trajectory of the limb from $200 \mathrm{msec}$ prior to impact up to $80 \mathrm{msec}$ after impact, as derived from the ensemble averages; the diagram corresponding to impact is marked with an asterisk. The (normalized) changes in the error of the vertical impulse responses of hand position $\left[\Sigma(y)^{2}\right]$ are plotted on the left of the corresponding set of stick diagrams. Maximum and minimum of hand error are aligned on the two broken vertical lines. The direction of movement is indicated by the arrows.

sition during catching. In fact, the largest variation of hand compliance coincides with limited and variable changes in hand position prior to impact. Thus, in all experiments maximum compliance occurs within the $200 \mathrm{msec}$ interval prior to impact and minimum compliance occurs at impact or very close to it. During that same time interval, hand position undergoes small changes in position: the hand is slightly raised in experiments $1-4,6$, and 8 , but it is slightly lowered in experiments 5 and 7 . By contrast, hand position undergoes a very large excursion during the first $80 \mathrm{msec}$ after impact, and yet hand compliance changes to only a limited extent during that same interval. Not only do the changes in hand compliance correlate poorly with the changes in hand position, but they also correlate poorly with the specific geometrical configuration of the limb. Thus, minimum hand compliance (close to the asterisk) can occur either with the wrist flexed (experiments 1, 4, 6, and 7) or with the wrist extended (experiments 2, 3,5, and 8).

\section{Discussion}

We shall first recapitulate the main findings of this article. In agreement with our previous results (Lacquaniti et al., 1991), we have shown a transient reversal of stretch reflex responses centered on impact time, leading to coactivation of antagonist muscles. We have then provided for the first time a quantitative description of the time-varying behavior of the compliance of a multijointed limb. We found that the performance error corresponding to the hand compliance in world coordinates is consistently minimized around impact, in coincidence with the transient reversal of stretch reflex responses. By contrast, the performance errors expressed in the angular coordinates of the joints have a more variable time course and are not minimized around impact.

In the following, we shall argue that the minimization of hand compliance is related to the reflex reversal and to the availability of an internal model of limb geometry.

\section{Functional significance of reflex reversal}

Reflex coactivation of antagonist muscles is a distinct mode of operation of the spinal circuits interposed between fast-conducting afferents and $\alpha$-motoneuron pools (Lacquaniti et al., 1991). It is centrally gated in lieu of reciprocal inhibition during the short time interval immediately preceding and overlapping the phase of dynamic interaction of the hand with the ball. Its 
significance is twofold. First, the time span $( \pm 60 \mathrm{msec}$ around impact) of the gating of the stretch reflex responses that are evoked by the torque motor perturbations overlaps the time of occurrence of the reflex coactivation that is evoked by ball impact at a latency of about $20 \mathrm{msec}$ (see Figs. 7, 8). Thus, this latter reflex coactivation might depend on the same central presetting mechanisms that are involved in the gating of the stretch reflex responses. If so, it follows that the CNS presets a coactivation response to the expected impulsive load of the impact with a safety margin of about $\pm 60 \mathrm{msec}$. As for the functional significance of the reflex coactivation evoked by ball impact, it has previously been argued that it contributes to the stabilization of the limb after catching (Lacquaniti and Maioli, 1989a).

However, there is an additional and more important significance of the transient reflex reversal. Clearly, the spinal network that is responsible for the stretch reflex responds to both external and internal inputs (cf. Loeb et al., 1989). In particular, the identification method of time-varying changes in the stretch reflex responses we used allows us to assess the manner in which afferent information normally generated during posture or movement is utilized in its control in the absence of large external perturbations (Soechting et al., 1981; Lacquaniti and Soechting, 1983). We hypothesize that the reversal of stretch reflex responses before impact indicates a transition between two distinct control modes: from a position control, based on reciprocal innervation, to a compliance control, based on coactivation. This hypothesis is supported by the following arguments.

A successful catch is contingent on an accuratc control of limb position to intercept ball trajectory at the right time (Sharp and Whiting, 1974). In parallel, this task also requires a control of limb compliance to absorb ball momentum during the dynamic interaction with the hand. Indeed, there is clear experimental evidence for both position control and compliance control. Anticipatory muscle activity is time locked to the estimated time to contact. Its mean amplitude scales linearly with the expected momentum at impact. This anticipatory activity is generally accompanied by limb flexion to meet the incoming ball.

Besides the anticipatory activity, the stretch reflex may also contribute to both position and compliance control. In particular, the reflex operating mode that involves reciprocal responses in antagonist muscles (as observed under basal conditions) has usually been interpreted in terms of position and velocity feedbacks (Stein, 1982; Agarwal and Gottlieb, 1984). As for the reflex mode involving coactivation, the nature of either the input error signal or the controlled variable remains to be determined. However, this mode should result primarily in a transient increase of joint stiffness (and viscosity), since the joint torques produced by antagonist muscles subtract but stiffnesses add. We speculate that this mode is aimed at tuning limb compliance for the optimal absorption of ball momentum right at the time of impact. A possible implementation of the switching between the two operating states would then consist in a network that changes synaptic weights so as to optimize a given performance criterion dynamically. Indeed, the performance error corresponding to the hand compliance in world coordinates was minimized in all experiments at impact, in coincidence with the reversal of stretch reflex responses. In a similar vein, Loeb et al. (1989) have suggested that the matrix of the feedback coefficients corresponding to the spinal connections of peripheral afferents involved in the control of cat posture and loco- motion may be state dependent, being adjusted to satisfy specific optimality criteria that can vary according to the task demands.

\section{Limb compliance}

The kinematic impulse responses describe the relation between torque input and position output. Thus, they provide a global measure of the limb compliance. This measure rests on no assumption other than linearity. The small amplitude pseudorandom perturbations employed in this study generally involve very limited nonlinearities (Soechting and Dufresne, 1980; Soechting and Lacquaniti, 1989). Indeed, we found that the linear impulse responses predict accurately the measured mechanical responses also under the present experimental conditions (see Materials and Methods).

This is the first study dealing with the time-varying properties of compliance in a multijointed limb. Consequently, no direct comparison can be made with other independent sets of data. However, the elbow impulse responses obtained under basal conditions are qualitatively similar to those described by Lacquaniti and Soechting (1986) using pseudorandom perturbations applied to the elbow and shoulder joints under stationary conditions.

In order to quantify the changes in limb compliance, we have used error criteria that describe concisely the amount of oscillation of the limb at different times during the catching task. We found that the error criterion corresponding to hand compliance in Cartesian coordinates is consistently minimized around impact time. Hand compliance is a global variable that reflects the dynamical state of the whole limb. In particular, it depends on the mean EMG activity, reflex behavior, and limb position (Mussa-Ivaldi et al., 1985; Kearney and Hunter, 1990). We shall consider each of these factors separately.

Mean EMG activity. Single-joint studies have demonstrated that angular stiffness and viscosity increase almost linearly with the level of mean muscle activity, due to an increasing number of engaged cross-bridges (Agarwal and Gottlieb, 1984; Kearney and Hunter, 1990). In the present experiments, the mean EMG amplitude at impact was greater than that under basal conditions in many cases, but it was smaller in some cases. Moreover, the temporal correlation between the changes in overall EMG activity and the changes in hand compliance was poor, the maximum in the former always lagging behind the minimum in the latter (see Figs. 7, 8).

Limb position. When subjects maintain a static limb posture, the hand stiffness depends strongly on the location of the hand in the external space; in other words, hand stiffness varies systematically with the geometrical configuration of the limb (Mussa-Ivaldi et al., 1985; Flash and Mussa-Ivaldi, 1990). In the present experiments, the instantaneous geometrical configuration of the limb varied appreciably and rapidly around impact time. However, the changes in hand compliance were poorly correlated with the changes in limb configuration (see Figs. 79).

Reflex reversal. Single-joint studies have shown that changes in the gain of the stretch reflex affect both angular stiffness and viscosity (Lacquaniti et al., 1982b; Akazawa et al., 1983). The reflex reversal we observed here does not simply represent a change in reflex gain, but is a change of the operational state of the system. Although we do not know the precise manner in which reflex reversal results in a modulation of limb compliance, its involvement is strongly suggested by the observation that 
reflex reversal was consistently correlated in time with the minimum in hand compliance, the former leading the latter by about 40 msec.

In sum, the reversal of stretch reflex responses is the only event whose timing is consistently related to the minimum in hand compliance. This observation does not imply that reflex reversal is the only causative factor involved in the minimization of hand compliance. In fact, this minimum must result necessarily from the interplay of all described neural, muscular, and geometrical factors. We hypothesize that neural output can effectively compensate for the influence of the geometrical configuration of the limb on the hand compliance, as demonstrated by the lack of correlation between the changes in hand compliance and the changes in limb position.

\section{Internal model of limb geometry}

In contrast with the single-joint case, in a multijointed limb the compliance is not simply a scalar quantity, but also has a directional character (Mussa-Ivaldi et al., 1985; Flash and MussaIvaldi, 1990). Here, we have seen that the minimization of limb compliance around impact time occurs in the Cartesian coordinates describing the position of the hand in world space. In particular, while the performance error in the horizontal direction remained small throughout, the error in the vertical direction was minimized at impact. The vertical corresponds to the direction of the dynamic interaction between the hand and the free-falling ball. By contrast, the performance errors expressed in the angular coordinates of the joints had a more variable time course and were not minimized around impact.

The dissociation of the performance errors, when expressed in the Cartesian coordinates of the hand and when expressed in the angular coordinates of the joints, has an important implication. The output of neural control processes is muscle activity that modulates directly muscle stiffness and viscosity, and therefore joint compliance. Thus, the minimization of hand compliance in world coordinates implics that the CNS is able to represent internally the intended hand compliance and to transform it into appropriate patterns of output muscle activities. However, hand compliance is a global variable that depends on both the pattern of muscle activities and the geometrical configuration of the limb. In other words, the same pattern of muscle activity may result in a very different value of hand compliance depending on the values of joint angles. Therefore, the transformation of intended hand compliance into the appropriate muscle activities requires an internal model of limb geometry. The results obtained about catching can then be interpreted as indicative of the fact that the stretch reflex is gated on the basis of an internal model of limb geometry.

This is a striking conclusion, because it leads to postulate a linkage between two different domains of neural control that are usually considered to be independent, remote from each other, namely the domain of the internal models and representations of limb geometry, on the one hand, and the domain of muscle reflex control, on the other hand. According to the prevailing views on the hierarchical organization of motor systems, the level of movement planning and the level of movement control represent two independent stages of processing, dealing with entirely different kinematic and dynamic variables. At the higher level of planning, limb movements are represented in the global terms describing the action of the end effector (e.g., the hand). By contrast, it is often assumed that the level of reflex control deals only with the local variables pertaining to a single muscle (e.g., muscle length, force, or stiffness).

The picture that comes out from this and other studies (Abbs and Gracco, 1984; Lacquaniti and Soechting, 1986), instead, is one in which the operation of the reflex control does also deal with global variables, much of the same nature as those involved in the process of trajectory formation. Indeed, we showed that gating of the stretch reflex is involved in the minimization of one such global variable, the hand compliance, based on an internal model of limb geometry.

An old quotation appears pertinent here: “The nervous system reflects the external world by creating an internal model of its environment. ... The idea of the reflex as a manner in which the images of the environment are derived from the model internal to the nervous system leads us to conclude that the reflex is by its nature an active process" (Bernstein et al., 1973).

It is conceivable that, in the course of the ontogenesis of the brain processes involved in the construction of motor acts, an isomorphism emerges epigenetically between the internal modcls of the body and spacc, on the one hand, and limb movement and its perception, on the other hand. The existence of these internal models allows a virtual, simulated exploration of the external environment and an anticipatory adaptation of the motor behavior to the demands posed by the environment before they actually occur.

The notion that the brain is endowed with fairly accurate internal models of limb geometry is well established and has long been subsumed under the neurological rubric of "body scheme" (cf. Gurfinkel and Levik, 1979). The body scheme is largely inborn and stable, but model parameters, such as the estimate of mass and length of the individual limb segments, can be recalibrated adaptively. An elegant study has recently demonstrated that proprioceptive information is essential to maintain an adequate internal model of the mechanical properties of the upper limb (Ghez et al., 1990; Gordon et al., 1990). Deafferented subjects (due to large-fiber sensory neuropathies) are unable to compensate for work space anisotropies in limb inertia and produce pointing errors that are direction dependent. Vision of the limb can partially correct these movement errors.

The role of internal models of limb geometry for the control of movement also emerges from other pointing studies. Thus, Lacquaniti et al. (1982a) showed that arm kinematics is unchanged when a pointer is used that doubles the effective length of the forearm. This indicates that the transformation of target location from the world coordinates into the angular coordinates of the joints incorporates information on the effective length of the limb segments (Lacquaniti, 1989).

Soechting and Flanders $(1989 a, b)$ have recently studied an arm pointing task in three dimensions and found that when pointing is performed to a remembered target in the absence of vision, there exist significant errors in distance only. These errors are accounted for by the specific nature of the transformation performed from the world coordinates of the target (as internally represented using visual information) to the intrinsic joint coordinates of kinesthetic representation of arm orientation. These sensorimotor transformations are predicated on accurate internal representations of limb geometry.

The neural substrates of the internal models of limb geometry still need to be elucidated. The neural representation of arm movement direction has been discovered in primate motor cortex and parietal area 5: this parameter is accurately encoded in 
the discharge of the neuronal population (Georgopoulous, 1988). Recent studies seem to suggest that neural representations of limb geometry may be found as low as at the level of premotoneuronal areas of the spinal cord (Fukson et al., 1980; Bizzi et al., 1991). Indeed, microstimulation of the upper and middle layers of the frog spinal cord gray matter, in conjunction with positioning of the leg in different work space locations, generates a force field directed toward a single point (Bizzi et al., 1991). This field is completely different from that generated by stimulation of the motoneurons. These results indicate that an internal model of limb configuration in space may be an emergent property resulting from an appropriate activation of the spinal premotoneuronal networks.

\section{References}

Abbs JH, Gracco VL (1984) Control of complex motor gestures: orofacial muscle responses to load perturbations of lip during speech. $J$ Neurophysiol 51:705-723.

Agarwal GC, Gottlieb GL (1977) Compliance of the human ankle joint. J Biomech Eng 99:166-170.

Agarwal GC, Gottlieb GL (1984) Mathematical modeling and simulation of the postural control loop. Part III. CRC Crit Rev Biomed Eng 12:49-93.

Akazawa K, Milner TE, Stein RB (1983) Modulation of reflex EMG and stiffness in response to stretch of human finger muscle. J Neurophysiol 49:16-27.

Arbib MA (1981) Perceptual structures and distributed motor control. In: Handbook of physiology, Sec 1, Vol 2, Pt 2 (Brookhart JM, Mountcastle VB, eds), pp 1449-1480. Bethesda, MD: American Physiological Society.

Åström KJ, Wittenmark B (1989) Adaptive control, pp 526. Reading, MA: Addison-Wesley.

Atkeson CG (1989) Learning arm kinematics and dynamics. Annu Rev Neurosci 12:157-183.

Bernstein N, Anochin PK, Sokolov EN (1973) Neurofisiologia e cibernetica (transl from Russian). Roma: Mecacci.

Bizzi E, Chapple W, Hogan N (1982) Mechanical properties of muscles: implications for motor control. Trends Neurosci 5:395-398.

Bizzi E, Mussa-Ivaldi FA, Giszter S (1991) Computations underlying the execution of movement: a biological perspective. Science 253: 287-291.

Cole KJ, Abbs JH (1988) Grip force adjustments evoked by load force perturbations of a grasped object. J Neurophysiol 60:1513-1522.

Davies WDT (1970) System identification for self-adaptive control, pp 380. London: Wiley.

Dufresne JR, Soechting JF, Terzuolo C (1978) Electromyographic response to pseudo-random torque disturbances of human forearm position. Neuroscience 3:1213-1226.

Feldman AG (1980) Superposition of motor programs. I. Rhythmic forearm movements in man. Neuroscience 5:81-90.

Flash T, Mussa-Ivaldi F (1990) Human arm stiffness characteristics during the maintenance of posture. Exp Brain Res 82:315-326.

Fukson OI, Berkinblit MB, Feldman AG (1980) The spinal frog takes into account the scheme of its body during the wiping reflex. Science 209:1261-1263.

Georgopoulos AP (1986) On reaching. Annu Rev Neurosci 9:147170.

Georgopoulos AP (1988) Spatial coding of visually guided arm movements in primate motor cortex. Can J Physiol Pharmacol 66:518526.

Georgopoulos AP, Kalaska JF, Massey JT (1981) Spatial trajectories and reaction times of aimed movements: effects of practice, uncertainty and change in target location. J Neurophysiol 46:725-743.

Ghez C, Gordon J, Ghilardi MF, Christakos CN, Cooper SE (1990) Roles of proprioceptive input in the programming of arm trajectories. Cold Spring Harbor Symp Quant Biol 55:837-847.

Gordon J, Ghilardi MF, Ghez C (1990) Deafferented subjects fail to compensate for workspace anisotropies in 2-dimensional arm movements. Soc Neurosci Abstr 16:445.25.

Gurfinkel VS, Levik YU (1979) Sensory complexes and sensorimotor integration. Human Physiol 5:269-281.
Hasan Z (1986) Optimized movement trajectories and joint stiffness in unperturbed, inertially loaded movements. Biol Cybern 53:373382.

Hildreth EC, Hollerbach JM (1987) Artificial intelligence: computational approaches to vision and motor control. In: Handbook of physiology, Sec 1, Vol 5, Pt 2 (Brookhart JM, Mountcastle VB, eds), pp 605-642. Bethesda, MD: American Physiological Society.

Hogan N (1984) Adaptive control of mechanical impedance by coactivation of antagonist muscles. IEEE Trans Autom Contr 29:681690.

Hogan N (1985) The mechanics of multi-joint posture and movement control. Biol Cybern 52:315-332.

Houk JC (1979) Regulation of stiffness by skeletomotor reflexes. Annu Rev Physiol 41:99-114.

Houk JC, Rymer WZ (1981) Neural control of muscle length and tension. In: Handbook of physiology, Sec 1, Vol 2, Pt 1 (Brookhart JM, Mountcastle VB, eds), pp 257-324. Bethesda, MD: American Physiological Society.

Humphrey DR, Reed DJ (1983) Separate cortical systems for control of joint movement and joint stiffness: reciprocal activation and coactivation of antagonist muscles. In: Motor control mechanisms in health and disease (Desmedt JE, ed), pp 347-372. New York: Raven.

Hunter IW, Kearney RE (1982) Dynamics of human ankle stiffness: variation with mean ankle torque. J Biomech 15:747-752.

Johansson RS, Westling G (1988) Programmed and triggered actions to rapid load changes during precision grip. Exp Brain Res 71:72-86.

Kearney RE, Hunter IW (1990) System identification of human joint dynamics. CRC Crit Rev Biomed Eng 18:55-87.

Lacquaniti F (1989) Control representations of human limb movement as revealed by studies of drawing and handwriting. Trends Neurosci 12:287-291.

Lacquaniti F, Maioli C (1987) Anticipatory and reflex coactivation of antagonist muscles in catching. Brain Res 406:373-378.

Lacquaniti F, Maioli C (1989a) The role of preparation in tuning anticipatory and reflex responses during catching. J Neurosci 9:134148.

Lacquaniti F, Maioli C (1989b) Adaptation to suppression of visual information during catching. J Neurosci 9:149-159.

Lacquaniti F, Soechting JF (1983) Changes in mechanical impedance and gain of the myotatic response during transitions between two motor tasks. Exp Brain Res [Suppl] 7:135-139.

Lacquaniti F, Soechting JF (1986) EMG responses to load perturbations of the upper limb: effect of dynamic coupling between shoulder and elbow motion. Exp Brain Res 61:482-496.

Lacquaniti F, Soechting JF, Terzuolo C (1982a) Some factors pertinent to the organization and control of arm movements. Brain Res 252: 394-397.

Lacquaniti F, Licata F, Soechting JF (1982b) The mechanical behavior of the human forearm in response to transient perturbations. Biol Cybern 44:35-46.

Lacquaniti F, Borghese NA, Carrozzo M (1990) Functional characteristics of reflex coactivation in man. Soc Neurosci Abstr 16:69.7.

Lacquaniti F, Borghese NA, Carrozzo M (1991) Transient reversal of the stretch reflex in human arm muscles. J Neurophysiol 66:939-954.

Loeb GE (1983) Finding common ground between robotics and neurophysiology. Trends Neurosci 6:203-204.

Loeb GE, He J, Levine WS (1989) Spinal cord circuits: are they mirrors of muscoloskeletal mechanics? J Motor Behav 21:473-491.

Mason MT (1981) Compliance and force control for manipulators. IEEE Trans Syst Man Cybern 11:418-432.

Mussa-Ivaldi FA, Hogan N, Bizzi E (1985) Neural, mechanical and geometric factors subserving arm posture in humans. J Neurosci 5: 2732-2743.

Sanes JN (1986) Kinematics and end-point control of arm movements are modified by unexpected changes in viscous loading. $J$ Neurosci 6:3120-3127.

Sharp RH, Whiting HTA (1974) Exposure and occluded duration effects in a ball catching skill. J Motor Behav 6:139-147.

Soechting JF (1988) Effect of load perturbations on EMG activity and trajectories of pointing movements. Brain Res 451:390-396.

Soechting JF, Dufresne JR (1980) An evaluation of nonlinearities in the motor output response to applied torque perturbations in man. Biol Cybern 36:63-71.

Soechting JF, Flanders M (1989a) Sensorimotor representations for 
pointing to targets in three-dimensional space. J Neurophysiol 62: 582-594.

Soechting JF, Flanders M (1989b) Errors in pointing are due to approximations in sensorimotor transformations. J Neurophysiol 62: $595-608$.

Soechting JF, Flanders M (1991) Deducing central algorithms of arm movement control from kinematics. In: Motor control: concepts and issues (Humphrey DR, Freund H-J, eds), pp 293-306. New York: Wiley.

Soechting JF, Lacquaniti F (1988) Quantitative evaluation of the electromyographic responses to multidirectional load perturbations of the human arm. J Neurophysiol 59:1296-1313.
Soechting JF, Lacquaniti F (1989) An assessment of the existence of muscle synergies during load perturbations and intentional movements of the human arm. Exp Brain Res 74:535-548.

Soechting JF, Dufresne JR, Lacquaniti F (1981) Time-varying properties of myotatic response in man during some simple motor tasks. J Neurophysiol 46:1226-1243.

Stein RB (1982) What muscle variable(s) does the nervous system control in limb movements? Behav Brain Sci 5:535-540.

Stein RB, Cody FWJ, Capaday C (1988) The trajectory of human wrist movements. J Neurophysiol 59:1814-1830.

Traub MM, Rothwell JC, Marsden CD (1980) A grab reflex in the human hand. Brain 103:869-884. 